\title{
LA CULTURA Y SUS FORMAS INSTITUCIONALES
}

\author{
Enrique López*
}

* Consejero del Consejo Nacional de la Cultura y las Artes 


\section{RESUMEN}

A partir de la ley que creó el Consejo Nacional de la Cultura y las Artes se modificó el esquema institucional referido a la cultura en Chile. Esta nueva estructura se suma a la tarea propia de las universidades, baluarte de su creación, mantención y transmisión. Ante esta nueva realidad, es necesario distinguir las competencias de cada uno de estos organismos, los que deben coordinarse y complementarse para lograr el bien cultural en un grado óptimo.

\section{ABSTRACT}

The law that created the National Council for the Arts and Culture modified the institutional scheme related to culture in Chile. The new structure joins universities in their own task, bastions of the creation, maintenance and transmission of culture. Faced with this new reality, it is necessary to distinguish each of the competencies of these organizations, which need to be coordinated and supplemented adequately in order to bring culture up to an optimum level. 


\section{LA CULTURA Y SUS FORMAS INSTITUCIONALES}

La cultura y la universidad

Hace años, un profesor en la Escuela de Derecho de la Universidad de Chile definía las instituciones como creaciones del hombre destinadas a satisfacer necesidades sociales, es decir, entes permanentes dotados de ciertas estructuras estables (pero, al mismo tiempo, dinámicas), y destinados a alcanzar sus propios objetivos. Si aceptamos esta definición, convendremos en que deben existir tantas instituciones como necesidades hay en el hombre, desde las más primitivas hasta las más sofisticadas: por ejemplo, deportivas, religiosas, de servicio a los demás, políticas propiamente tales, reivindicativas y, por supuesto, culturales.

La cultura es la dimensión más propiamente humana; es la aventura del cultivo de lo material, de la naturaleza y del espíritu. Según Christopher Dawson, la cultura “...es la forma o estilo de la vida social (the way of life) de una comunidad humana que se adapta a un medio ambiente particular; resulta del trabajo de sucesivas generaciones y se transmite de edad a edad, y también implica un cierto grado de especialización y canalización de las energías humanas y sociales, conforme a pautas comúnmente establecida y aceptadas" ${ }^{1}$.

"El [elemento] sicológico racional, es decir, el pensamiento, actividad específica del ser humano, lo libera de la dependencia ciega de las condiciones materiales que caracteriza a las formas inferiores de vida; este factor influye mediante el lenguaje, que es el elemento

1 Dawson, Christopher, Historia de la Cultura Cristiana (compilación), Traducción e introducción de Heberto Verduzco Hernández, Breviarios, Fondo de Cultura Económica, México, 1997, p. 15. 
más fundamental de la cultura, pues es el instrumento de expresión y comunicación del pensamiento y de la relación e interacción entre los hombres [...] Toda la experiencia humana, tanto individual como colectiva, se desarrolla con el apoyo del símbolo lingüístico [...] en el lenguaje se almacena, intercomunica, enriquece y transmite el patrimonio cultural de la comunidad" 2 .

La cultura es una tarea que se ha dado permanente a lo largo de la historia de la humanidad y está presente dondequiera que dirijamos nuestra mirada ${ }^{3}$, desde las tosquedades iniciales a las creaciones clásicas. "Es, en sentido estricto, un artificio del logos, es una obra exclusivamente humana. En un astro deshabitado no hay cultura. Sólo naturaleza" ${ }^{4}$.

Cuando ya habían pasado miles de años desde la aparición del hombre sobre la tierra; cuando ya habían desfilado numerosas sociedades, culturas y civilizaciones -primarias y esplendorosas, tribus e imperios, dioses, adivinos y oráculos, dialectos y lenguas-, la cultura propiamente tal encontró un lugar donde instalarse en aquella magnífica institución que nos legara el mundo medieval: la universidad. En ella se radicarán el conocimiento y la búsqueda de la verdad y la ciencia, su acrecentamiento y su comunicación.

La universidad, lugar de encuentro de maestros y escolares, según el Libro de las Siete Partidas, es propiamente el espacio en que todos intentan aprender (también los profesores) y a desarrollar la inteligencia en un nivel superior, con método y de manera científica.

Buena parte de la cultura se radica en esta institución. Pero, además, desarrolla otras tareas definitorias de su naturaleza: la conservación y la generación del conocimiento transformado en ciencia, como disciplina racional del intelecto.

2 Ibíd, p. 16.

3 Herrera Cajas, Héctor, Dimensiones de la responsabilidad educacional, Editorial Universitaria, Universidad Metropolitana de Ciencias de la Educación, Colección Fuera de Serie, Santiago de Chile, 1988, pp. 34-35.

4 Fernández de la Mora, Gonzalo, "El Compromiso Cultural del Estado", en Razón Española, $\mathrm{N}^{\circ} 117$, enero, febrero 2003, Madrid, España, pp. 12-29. 
En nuestra época, las sociedades han exigido mucho de la universidad y han esperado mucho de ella, quizás demasiado. Han aguardado por cultura, sentido crítico, discernimiento sobre la propia sociedad, buenos profesionales, investigación científica, tradición e innovación, cambios metodológicos, nuevas tecnologías, entre otros aportes. Entonces, es bueno preguntarse acerca de cuáles son las cosas esenciales que le corresponden sólo a ella, porque, como afirmaba Jorge Millas, la "lista de cosas esenciales tiende a crecer en desmesura", y, entonces, en esa desproporción se divisa paralelamente una evasión hacia otras dimensiones que pueden ser adjetivas y no sustantivas a ella. Pudiera darse la paradoja de que la universidad se preocupe de muchas cosas y poco de las que le son privativas.

Es propio de la universidad la investigación, para enseñar fructíficamente. Hay corporaciones que existen sólo para hacer investigación, pero que no enseñan. La universidad también investiga, porque, además del valor intrínseco que esta búsqueda posee, debe transmitir el nuevo conocimiento a sus alumnos y a la población en general. Si ella investiga, todo lo demás -como lo señala un distinguido profesor- viene necesariamente por añadidura ${ }^{5}$.

Suele señalarse como propio de estas corporaciones la tríada: docencia, investigación y extensión. Conviene, entonces, detenerse sobre todo en este último término para realizar un análisis crítico.

Antes de ello, dejemos establecido que, con lo importante que puede ser la universidad como un vehículo de cultura, no es la única institución que la refleja. Es cierto que en los claustros universitarios hay más selección y refinamiento, pero la cultura en su rica complejidad numerosas veces huye de lo clásico y se desarrolla al margen de la mirada exigente de la educación superior. Con todo, lo cierto -qué duda cabe- es que la universidad tiene una inexcusable responsabilidad cultural, pues, a través suyo, se refleja a la comunidad de manera más pensada y trabajada.

5 Guzmán Brito, Alejandro, "La Universidad Investigadora", en Academia, No 15, 1987, p. 26. Citado en Rojas S. G., Textos fundamentales sobre la Universidad, Editorial Universitaria, Santiago de Chile, 1988, p. 59. 
La universidad y la extensión

He señalado que es propio de la universidad investigar y enseñar sus resultados en sus aulas, pero afirmo que no es propiamente sustantivo de ella la extensión. Sin embargo, cuando es más fiel a sí misma, cuando cumple lo suyo de manera exigente, tiene algo que extender a la comunidad, tiene frutos que mostrar, y esto es lo que propiamente se llama extensión.

La extensión es diferente de la mera difusión. Así lo ha señalado lúcidamente el profesor Alejandro Guzmán, al señalar que “...toda actividad de extensión que no sea la de difundir resultados científicamente alcanzados en el seno de la universidad no es, desde un punto de vista funcional, 'extensión universitaria' y tan sólo orgánicamente podría ser denominada 'extensión de la universidad'. Cuando ésta publica un libro de un profesor suyo está haciendo extensión universitaria, pero cuando ofrece un film comercial no hace extensión universitaria; en ese caso, la universidad hace extensión no

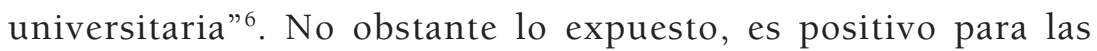
corporaciones de educación superior, y para la comunidad, que aquellas, además de extensión, hagan difusión.

Dicho rudamente, a algunas casas de estudios superiores sólo les resta difundir porque no tienen nada que extender. Algunas de ellas, pese a su nula o escasa labor investigadora, son interesantes instituciones difusoras; desde su privilegiada situación en el mundo de la educación y la cultura pueden colaborar subsidiariamente, junto a otras instituciones-como, por ejemplo, las corporaciones culturales municipales-, a mantener y difundir variadas expresiones culturales. Esto explicaría que algunas universidades que no desarrollan bajo ningún aspecto las artes plásticas, ni en docencia ni en investigación, realicen cada cierto tiempo estupendas exposiciones; o que respetables universidades autónomas de reciente creación, no obstante no desarrollar ningún programa académico-musical, mantengan sorprendentes ciclos de conciertos. Sea cual sea el caso, el universo de sujetos a los cuales se dirigen estas actividades de extensión o de difusión es muy pequeño, localizado y muchas veces también muy especializado.

6 Ibíd, p. 26. 
Además, cabe advertir -y fluye de lo expuesto- que a la extensión universitaria, cuando es tal, se le debe exigir estándares de calidad que a la simple difusión no se le exigiría, pues, como ya se afirmó, su naturaleza y fines son distintos. Asimismo, es necesario señalar que, numerosas veces, la extensión es cultura, otras ciencia pura y otras expresión profesional; pero, cualquiera sea el caso, debe realizarse toda extensión que sea digna de ese nombre. Igualmente, sin esta tarea -que es deseable- la universidad no deja de ser tal, pues, como afirmase Jorge Millas, "en rigor la extensión es función de complemento, y no esencial"'.

Pero el apasionante mundo de la cultura -entendida en términos generales- tiene tal complejidad y dimensiones, que, numerosas veces, huye de las formas que pretenden enmarcarla en estructuras institucionalizadas. La cultura es notablemente real; se podría afirmar, casi sin temor a errar, que gran parte de ella pasa al margen de las universidades.

La cultura y la institucionalidad emergente

La Constitución Política impone una serie de deberes al Estado, a la comunidad nacional y a las personas. Al primero le asigna muchas tareas (quizás demasiadas) entre las cuales no suele destacarse fomentar el desarrollo de la educación en todos sus niveles, estimular la investigación científica y tecnológica, la creación artística y la protección e incremento del patrimonio cultural de la nación ${ }^{8}$.

Tradicionalmente, en Chile, el Estado ha cumplido este último mandato (aun antes de la Constitución de 1980) a través del Ministerio de Educación y sus divisiones dependientes, entre las cuales se destaca la Dirección de Bibliotecas, Archivos y Museos - una de las unidades en que con mayor propiedad descansan la conciencia y tradición nacionales-, otros ministerios y, naturalmente, las universidades. Sin embargo, no debe perderse de vista que éstas últimas son entes

7 Millas, Jorge, "Misión de la Universidad frente a las Ciencias y las Artes", en Idea y Defensa de la Universidad, 1981, p. 111. Citado en Rojas Sánchez, G., Textos fundamentales sobre la Universidad, Editorial Universitaria, Santiago de Chile, 1988, p. 79.

8 Constitución Política de la República de Chile, Artículo 19, $\mathrm{N}^{\circ} 10$, inciso $5^{\circ}$, Editorial Jurídica de Chile, Santiago de Chile, 2003, Ley No 19.891, 23 de agosto de 2003, Artículo $2^{\circ}$. 
autónomos, académica, funcional y económicamente hablando. Ellos extienden sus conocimientos, descubrimientos e investigaciones si tienen algo que extender; pero definen, priorizan y determinan, en virtud de su propia facultad de autodeterminación, qué aspectos culturales desean extender; a la sociedad. Muchas veces los académicos seleccionan aspectos de su interés y para grupos especializados que comprenden esos códigos muy parciales.

A partir de la ley $\mathrm{N}^{\circ}$ 19.891, del 23 de agosto de 2003, la forma de enfrentar el cumplimiento del mandato constitucional se ha modificado, al crearse el Consejo Nacional de la Cultura y las Artes, como un servicio público autónomo, descentralizado y territorialmente desconcentrado, con personalidad jurídica y patrimonio propio. A partir de esta nueva realidad legislativa, la situación ha cambiado, pues emerge otra institucionalidad orgánica directamente relacionada con el Ejecutivo. Esta es una fórmula reciente destinada a apoyar el desarrollo de las artes y la difusión de la cultura, contribuyendo a conservar, incrementar y poner al alcance de las personas el patrimonio cultural de la nación y promover la participación de éstas en la vida cultural del país.

Ahora, entonces, existe una ordenación institucional principiante, novel, cuyo objetivo preciso es este bien tan preciado que es la cultura. Bien -cabe advertir- que existe de todas maneras en sí mismo, independiente de sus formas oficiales, y que debe ser descubierto, respetado e impulsado donde éste se encuentra, desde las cantoras de los rodeos hasta las decenas de pueblos rurales detenidos en el tiempo con sus arquitecturas, pequeños museos y espacios añosos; desde el aprovechamiento y renovación de espacios urbanos, a las iglesias y sus pinturas, el rescate de canciones originales, películas y obras de teatro que deben conservarse y reproducirse entre tantas manifestaciones.

Todos ellos y muchos otros constituyen bienes invaluables, individuales, grupales y sociales, pero parte indivisa de lo que conocemos como "bien común", que consiste en crear las condiciones sociales que permitan a todos y a cada uno de los integrantes de la comunidad nacional su mayor realización espiritual y material posible ${ }^{9}$.

9 Ibíd, Artículo $1^{\circ}$, inciso $4^{\circ}$. 
En la consecución de este bien están comprometidas la suerte e identidad de la nación.

En la concreción de este objetivo siempre es posible hacer mejor las cosas. No hacerlo, o no hacerlo suficientemente o eficientemente, significa desatender también lo esencial y permanente; pero, además, significa impulsar un desarrollo inconsistente que despliega casi todas sus energías en lo específicamente económico-social, que, es cierto, tiene demandas inmediatas sobre el sistema, en desmedro de aquellas actividades humanas más humildes y más espléndidas de la cultura. Un desarrollo que no atienda con verdad y con autenticidad a la dimensión de la cultura trunca ese desarrollo; más aún, se puede afirmar que no es auténtico desarrollo.

\section{Extensión y difusión}

Por lo expuesto y considerando la nueva realidad institucional de la cultura, parece importante reflexionar sobre las competencias de las instituciones comprometidas en esta actividad.

Por de pronto, es útil establecer de inmediato que, sean éstas cuáles fueren y sobre qué actúen, ambas dimensiones: el Consejo Nacional de la Cultura y las Artes y la universidad concurran a mantener y desarrollar un solo bien: la cultura.

Cada una de estas instituciones -las universidades y el Consejo Nacional de la Cultura y las Artes, recientemente creado, donde también hay representantes de las universidades estatales y universidades privadas y autónomas-, con sus respectivos recursos, sus capacidades y energías, concurren a un mismo objetivo, pero que cubre ámbitos y dimensiones distintas. Las universidades realizan lo propio, es decir, extensión y subsidiariamente difusión, y el Consejo -directamente-, difusión, tareas de estudio, ejecución y evaluación de políticas culturales, funciones de estudio e investigación de la actividad cultural del país, correspondiéndole también velar por la participación de personas y asociaciones, fomentar aptitudes de gestión, impulsar la construcción y habilitación de infraestructura, desarrollar la cooperación, asesoría técnica e interlocución, y establecer vínculos de coordinación y colaboración con todas las reparticiones 
públicas que, sin formar parte del Consejo ni relacionarse directamente con éste, cumplan también funciones en el ámbito de la cultura ${ }^{10}$.

Al parecer una de las claves del actual esquema institucional se encuentra en este último aspecto. Las manifestaciones de la cultura son muchas y con grandes necesidades, y los recursos para satisfacerlas son escasos. Esta premisa básica de la economía se aplica con fuerza en esta área. De manera que la lógica impone el mayor aprovechamiento de los capitales disponibles. El mandato de la realidad, entonces, debe expresarse en la máxima colaboración y eficaz complementariedad entre las instituciones ${ }^{11}$.

El país y su cultura pueden esperar mucho de la difusión de unos y la extensión de otros.

10 Ibíd, Artículo 19, $\mathrm{N}^{\circ} 10$, inciso $5^{\circ}$.

11 En términos concretos, y en la línea de todo lo expuesto, es oportuno destacar un ejemplo de colaboración en la extensión cultural: el acuerdo suscrito entre el Supremo Gobierno, a través del Consejo Nacional de la Cultura y las Artes, y la Universidad de Chile para la reconstrucción del Museo de Arte Contemporáneo. 